\title{
Integrated Filtered Feedback Tunable Laser using Double-Ring-Resonator-Coupled Filter
}

\author{
Shinji Matsuo ${ }^{1}$, Toru Segawa ${ }^{1}$, Takaaki Kakitsuka ${ }^{1}$, Tomonari Sato ${ }^{1}$, Ryo Takahashi ${ }^{1}$, \\ Hiroyuki Suzuki ${ }^{1}$, Boudewijn Docter ${ }^{2}$, Fouad Karouta ${ }^{2}$, and Meint Smit ${ }^{2}$ \\ 1: NTT Photonics Laboratories, NTT Corporation, 3-1 Morinosato-Wakamiya, Atsugi, \\ Kanagawa, 243-0198, Japan \\ 2: COBRA Research Institute, Technical University of Eindhoven, \\ P.O. Box 127, 5600 MB Eindhoven, The Netherlands
}

\begin{abstract}
A novel integrated filtered feedback tunable laser consisting of a Fabry-Perot laser and integrated filtered feedback section is proposed and demonstrated. The device exhibited a $24-\mathrm{nm}$ tuning range and $1-\mathrm{GHz}$ thermal frequency drift.
\end{abstract}

\section{Introduction}

Optical packet switching systems require widely tunable lasers with a fast tuning speed because these lasers are used with an arrayed waveguide grating (AWG) filter in a wavelength routing switch, which is a key component of the systems. With this switch, the destination port can be controlled by changing the lasing wavelength. The monolithic integration of tunable lasers and an AWG filter is desired for size and cost reduction. With this in mind, we have developed a double-ring-resonator-coupled tunable laser [1] (DRR-TL) because it has a small, simple structure and is suitable for monolithic integration with other components. As an example, we have also realized the monolithic integration of a semiconductor optical amplifier (SOA) based wavelength converter with a DRR-TL [2]. However, an important issue remains with regards switch performance, namely the reduction of the thermal frequency drift seen after changing the lasing wavelength, as this frequency drift increases the crosstalk to the neighboring output channel of the AWG filter.

To overcome this problem, we propose and demonstrate a novel tunable laser that we call an integrated filtered feedback tunable laser or IFF-TL. The IFF-TL consists of a Fabry-Perot laser diode (FP-LD) monolithically integrated with a filtered feedback section. The device has a 24-nm tuning range with a switching time of less than $5 \mathrm{~ns}$. The thermal frequency drift is only $1 \mathrm{GHz}$.

\section{Device structure and operating principle}

Fig. 1 shows a photograph of the fabricated tunable laser, consisting of an FP-LD and filtered feedback section, and the operating principle. The cavity of the FP-LD is defined by an etched gap mirror and a cleaved facet. The filtered feedback section consists of an SOA, a phase control region, and a double-ring-resonator-coupled tunable filter. When there is no feedback section, the output light from the FP-LD has multiple spectral peaks depending on the laser cavity length as shown in Fig. 1(b). The feedback section has a series connection of two ring resonators with different FSRs to provide a wide tuning range filter, as shown in Fig. 1(c). The feedback section selects one channel from the multiple lasing peaks of the FP-LD output and then the filtered light is re-injected (feedback) into the FP-LD, as shown in Fig. 1(d). Thus, the FP-LD operates as a single-mode laser and the lasing frequency can be selected by controlling the resonant frequency of the ring resonators. The re-injected light power is controlled by the reflectivity of the facet and the gain of the SOA. The switching power of a side-mode injection laser depends strongly on the detuning from the

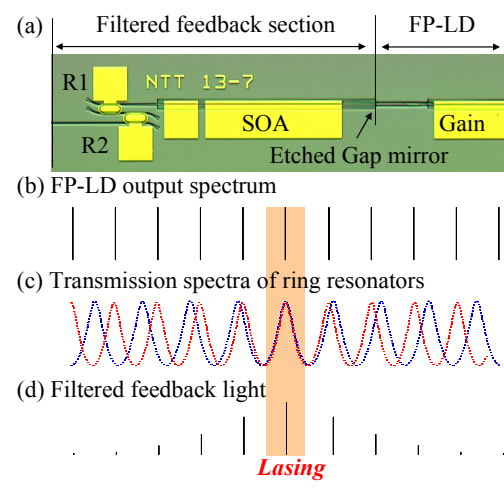

Fig. 1. Photograph of the proposed tunable laser and operating principle

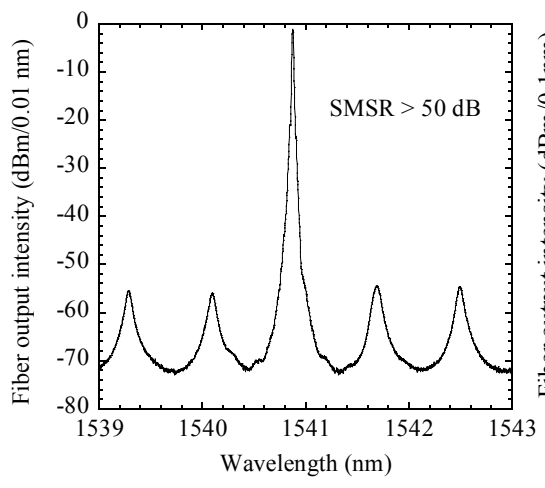

Fig. 2. Lasing spectrum

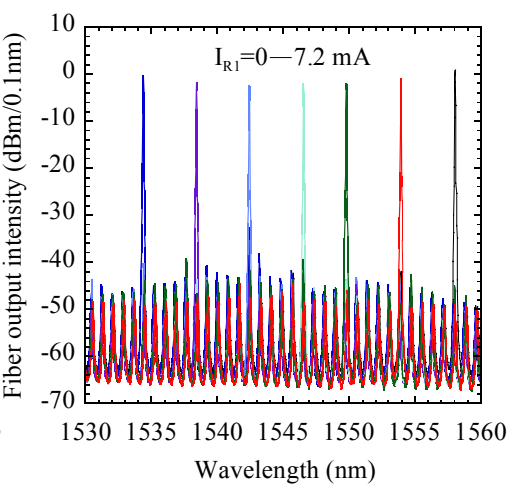

Fig. 3. Tuning characteristics 
side-mode wavelength and increases with wavelength detuning [3, 4]. The proposed tunable laser can thus switch the lasing wavelength with low power because the re-injected light originates from the FP-LD itself. This low injection power is important for preventing multiple cavity mode operation in the proposed laser.

\section{Device characteristics}

The device was monolithically fabricated using a stacked-layer structure [2]. The gain, phase control regions, and the SOA have a shallow-ridge waveguide structure, whereas the ring resonator has a deep-ridge waveguide structure. The device is $1.4 \mathrm{~mm} \times 350 \mu \mathrm{m}$ in size, including the bonding pads. The lengths of the gain region and SOA are 200 and $400 \mu \mathrm{m}$, respectively. The FSRs of R1 and R2 were 500 and $535 \mathrm{GHz}$, respectively. The etched gap mirror had about $30 \%$ reflectivity, with $0.75-\mu \mathrm{m}$ gap.

Fig. 2 shows the lasing spectrum of the laser when the device was $\mathrm{CW}$-operated at room temperature. The gain current was $80 \mathrm{~mA}$, the SOA current was $30 \mathrm{~mA}$, the current to R1 was $0 \mathrm{~mA}$, and the current to R2 was $2.1 \mathrm{~mA}$. One cavity mode was selected from $100-\mathrm{GHz}$ periodic Fabry-Perot modes. The low feedback light intensity suppresses the operation in multiple cavity modes other than Fabry-Perot modes. The side-mode suppression ration (SMSR) was more than $50 \mathrm{~dB}$. Fig. 3 shows the tuning characteristics. In this experiment, the gain current and the SOA current were the same as mentioned above. The current to the phase control region was kept constant at $0 \mathrm{~mA}$. When the injection current of R1 was changed from 0 to $7.2 \mathrm{~mA}$ and the injection current of R2 was controlled in the 0.5 to $0.8 \mathrm{~mA}$ range, the device exhibited a $24-\mathrm{nm}$ tuning range. As shown by these results, the integrated filtered feedback section can select one lasing mode from the Fabry-Perot modes.

Fig. 4 shows the dynamic response of the device. In this experiment, the current injected into R1 was changed from 0 to $6.1 \mathrm{~mA}$ at an interval of $50 \mathrm{~ns}$. The device provides fast and stable wavelength switching within 5 ns. Fig. 5 (a) and (b) show the thermal frequency drift over a longer time span for the proposed IFF-TL and the DRR-TL, respectively. The DRR-TL has the same structure as the feedback section of the proposed laser. In these experiments, the gain currents were 123.5 and $127 \mathrm{~mA}$ for the IFF-TL and DRR-TL, respectively, and the modulation currents into R1 were $8 \mathrm{~mA}$ for both lasers. The thermal frequency drift of the IFF-TL was less than $1 \mathrm{GHz}$ whereas that of the DRR-TL was about $7 \mathrm{GHz}$. The thermal frequency drift is caused by the longitudinal mode change in the laser cavity. In the DRR-TL, the refractive index change caused by the thermal transient directly changes the longitudinal mode. However, in the IFF-TL, the longitudinal mode of the laser is mainly determined by the FP-LD, and therefore the longitudinal mode change is extremely small.

\section{Conclusion}

We proposed and demonstrated an integrated filtered feedback tunable laser for the first time. The lasing wavelength of the FP-LD can be tuned by selecting the re-injected light wavelength from the feedback section. The device exhibited a wide tuning range of $24 \mathrm{~nm}$ and an extremely small thermal frequency drift of $1 \mathrm{GHz}$. These results indicate that the proposed tunable laser is extremely promising for constructing a fast wavelength routing switch.

\section{References}

1. S. Matsuo et al., "Widely tunable laser using microring resonators," ISLC2006, pp. 21-22, 2006.

2. T. Segawa et al., "Monolithically Integrated Filter-Free Wavelength Converter with Widely Tunable Double-Ring Resonator Coupled Laser," IPRM2008, TuA1.4, 2008.

3. K. Inoue, et al., "Bistability and Waveform Reshaping in a DFB-LD with Side-Mode Light Injection," IEEE Photon. Technol. Lett., vol. 7, no. 2, pp. 164-166, 1995.

4. S. Matsuo et al., "Optical Flip-flop Operation Using a DBR Laser," CLEO Pacific Rim 2007, pp. 1385-1386, 2007.

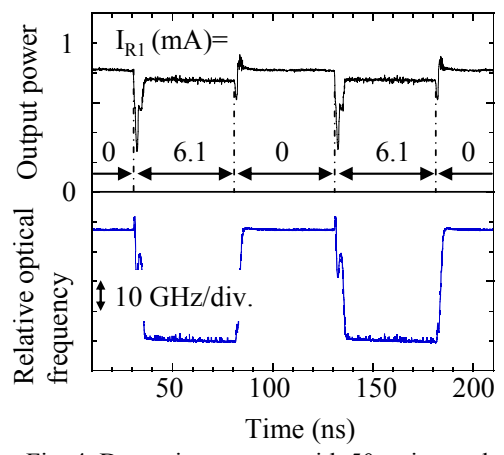

Fig. 4. Dynamic response with $50 \mathrm{~ns}$ intervals.
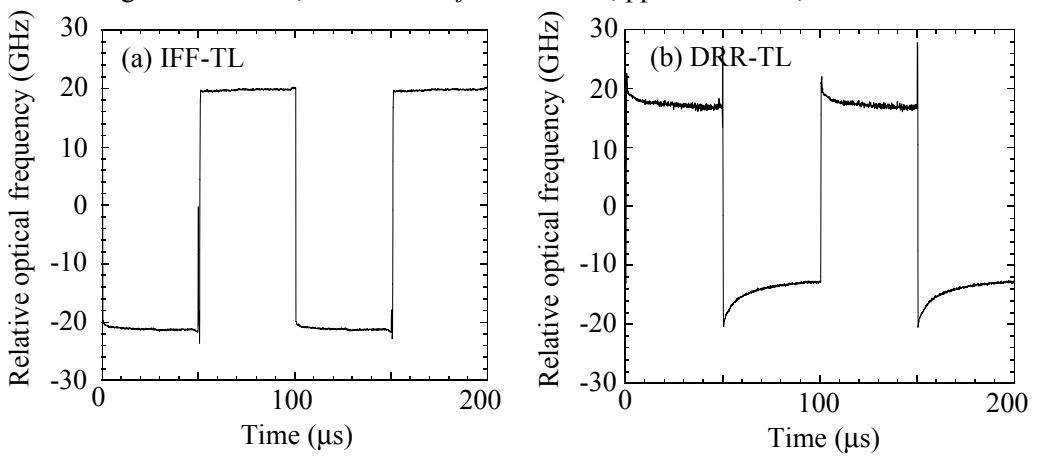

Fig. 5. Experimental results showing thermal frequency drift of (a) IFF-TL and (b) DRR-TL. 